\title{
Lesson for the clinical nephrologist: Kidney transplant, COVID-19 and pregnancy
}

\author{
Sarah Gleeson ${ }^{1,2}\left(\mathbb{C} \cdot\right.$ Muna Noori $^{2} \cdot$ Liz Lightstone $^{1,3} \cdot$ Philip Webster $^{1}$
}

Received: 5 August 2020 / Accepted: 28 October 2020 / Published online: 12 November 2020

(c) Italian Society of Nephrology 2020

Keywords Pregnancy $\cdot$ Obstetric nephrology $\cdot$ Kidney transplant $\cdot$ COVID-19

\section{Case report}

In April 2020, a 29-year-old nulliparous Asian woman, in a COVID-19 endemic area (London, United Kingdom), presented to a maternity hospital at 30 weeks gestation with 5 days of dry cough and shortness of breath. Further questioning revealed, anosmia, nausea, poor appetite and decreased fluid intake. She reported normal fetal movements.

Her past medical history was significant for end stage kidney disease of unknown aetiology. She had undergone 4 years of haemodialysis before receiving a deceased donor kidney transplant 18 months prior to pregnancy. Before this admission, her pregnancy was managed by the joint obstetric-renal clinic and had been uncomplicated. She was on tacrolimus monotherapy maintenance immunosuppression following alemtuzumab induction.

On examination she was tired and short of breath at rest. She was speaking in full sentences and not using her accessory muscles of respiration. Her temperature was $38^{\circ} \mathrm{C}$, heart rate 125 beats per minute $(\mathrm{bpm})$, respiratory rate was between 28-32 breaths per minute (Bpm) and her oxygen saturations were $97 \%$ on room air. Blood pressure was $123 / 73 \mathrm{mmHg}$. She had right basal crepitations. Her abdomen was non-tender and the fundal height was appropriate

Sarah Gleeson

sarah.gleeson2@gmail.com

1 Imperial College Renal and Transplant Centre, Imperial College NHS Trust, London, UK

2 Department of Obstetrics and Gynaecology, Queen Charlottes and Chelsea Hospital, Imperial College NHS Trust, London, UK

3 Department of Immunology and Inflammation, Faculty of Medicine, Centre for Inflammatory Disease, Imperial College London, London, UK for gestation. Her cardiotocograph showed fetal tachycardia at $175 \mathrm{bpm}$ (the upper limit of normal being $160 \mathrm{bpm}$ ).

Given the clinical setting, COVID-19 was immediately suspected with influenza and bacterial pneumonia as differential diagnoses. She was placed in respiratory isolation, and a nasopharyngeal COVID-19 swab was taken, and routine blood tests and a chest $\mathrm{x}$-ray were carried out. Her blood tests showed a neutrophil count of $15.3 \times 10^{9}(4.2-11.2)$, lymphocytes of $1.5 \times 10^{9}(1.1-3.6)$ and CRP of $65.9 \mathrm{mg} / \mathrm{L}(0-5)$. Creatinine was $86 \mathrm{umol} / \mathrm{L}$ from an in-pregnancy baseline of 60umol/L. Her chest X-ray showed multiple opacities in keeping with COVID-19. Her nasopharyngeal swab later confirmed the presence of viral SARS-COV-2 RNA. Inflammatory cytokine assays were not checked. She was started on $5 \mathrm{~L}$ of oxygen via nasal prongs and co-amoxiclav, clarithromycin and $0.9 \%$ saline intravenously.

Given the initial maternal tachycardia and tachypnoea, and the fetal tachycardia, there were concerns that delivery may need to be expedited. She was started on intravenous magnesium sulphate for fetal neuroprotection and intramuscular betamethasone to promote fetal lung maturation. After initial treatment with oxygen, fluid resuscitation and betamethasone, both maternal signs (heart rate $100 \mathrm{bpm}$, respiratory rate $22 \mathrm{Bpm}$, oxygen saturation $99 \%$ ) and fetal condition (resolution of the uncomplicated fetal tachycardia) improved over the subsequent $6 \mathrm{~h}$. The obstetric team discussed her care with the obstetric physicians and renal team and a decision was made to delay delivery and monitor closely. Given the known increased thrombotic risk associated with active COVID-19 infection, she was started on prophylactic low molecular weight heparin. She gradually improved over the next few days. Her requirement for supplemental oxygen stopped on day three and she was discharged on day 5 . Her trough tacrolimus levels were within our target range (5-8umol/1) and her dose was not changed. 
Following discharge, she was followed up in the renal obstetric clinic via a combination of virtual and face-to-face visits for the remainder of her pregnancy. She recovered completely from her COVID-19 symptoms. Her creatinine improved to 70-75 umol/L. Her growth scans showed continued normal growth velocity with normal umbilical artery dopplers. The rest of her pregnancy was uncomplicated.

As she recovered completely, had a normally grown baby and no other complications it was decided to aim for a normal delivery. She had an induction of labour at $40+5$ weeks for post-maturity. She required assisted delivery with a Ventouse cup, which was complicated by a $1 \mathrm{~L}$ post-partum haemorrhage, and a blood transfusion. She delivered a healthy female infant weighing 2900 grams (14th customised birth centile). The neonate was not tested for viral SARS-COV-2 RNA or SARS-COV-2 antibodies.

\section{Lessons for the clinical nephrologist}

- This patient's pregnancy was complicated both by her kidney transplant and maintenance immunosuppression and then by COVID-19 infection requiring admission and supplemental oxygen. The combination of COVID-19 infection in a pregnant kidney transplant recipient has not previously been reported.

- Recent emerging infections have had important effects on pregnancy [1]; both the 2009 H1N1 influenza outbreak and the 2002 severe acute respiratory syndrome (SARS) outbreak caused increased morbidity and mortality in pregnant women compared to the general population, and more recently Zika virus was associated with congenital abnormalities.

- As COVID-19 emerged there were concerns about its potential effects in pregnancy [1]. We now know that pregnant women do not appear to be more susceptible to getting COVID-19 [2]. Compared with age-matched controls, COVID-19 infection during pregnancy is associated with higher rates of hospitalisation and ICU admission but not mortality [3]. A large UK population-based study of pregnant women with COVID-19 admitted to hospital found $10 \%$ required ICU admission and $1 \%$ died [4]. COVID-19 infection was associated with pre-term delivery in $27 \%$ and caesarean delivery in $60 \%(16 \%$ for maternal respiratory compromise, $44 \%$ for obstetric indications). Five $\%$ of newborns born to mothers with COVID-19 tested positive for coronavirus, half in the 12 hours post-delivery [4].

- There have also been concerns about kidney transplant recipients and COVID-19, so much so that they have been placed in the 'extremely vulnerable' group by the United Kingdom government and advised to 'shield'essentially stay indoors apart from essential medical visits. There are several reports now of kidney transplant patients presenting with a mixture of mild, moderate or severe disease, with high rates of ICU admission in $30-57 \%$ and a high mortality rate of $10-28 \%[5,6]$. Over the short timeline of COVID-19, whether to reduce or stop immunosuppression and which immunosuppression to stop have been the subject of debate. Some authors have advocated for reduction or cessation of calcineurin inhibitors (CNIs) while others have suggested they may have a protective effect ${ }^{7}$. Studies of kidney transplant recipients with COVID-19 report high rates (62-86\%) of immunosuppression reduction (both CNIs and antiproliferative agents) especially in hospitalised or severely ill patients [5, 6]. More recent guidelines suggest stopping antiproliferative agents and consider reducing CNIs, especially in hospitalised or severely ill patients [7]. No rejection episodes have been reported in those who have reduced immunosuppression [6].

- Ideally, women with kidney transplants should 'shield' effectively during pregnancy to reduce the risk of infection. However, The Royal College of Obstetricians and Gynaecologists' guidelines on managing pregnancy during the COVID-19 pandemic suggest that although women with kidney transplants should ideally shield, they will need regular face-to-face visits to monitor graft function, drug levels and maternal/fetal complications [8]. As they require face-to-face visits, it suggests these patients be seen at the end or start of clinic hours, or outside of regular clinics, and that they should be isolated on attendance to minimise the risk of infection.

- COVID-19 needs to be high on the differential for those with typical (cough, fever, shortness of breath) and less typical (anosmia, diarrhoea, myalgia) symptoms. Once suspected, a test should be performed as soon as possible and the patient should be isolated [2]. Given the overlapping symptoms, screening for influenza should be considered.

- Active COVID-19 infection/respiratory compromise needs to take precedence. Oxygen, continuous positive airway pressure or mechanical ventilation should be considered, as appropriate. Delivery should be considered for obstetric indications or if there is maternal compromise which may benefit from delivery [2]. Venous thromboembolism (VTE) prophylaxis is important in all pregnant in-patients and especially so in COVID-19 [2].

- This patient presented before the results of the RECOVERY trial were known. Her care was supportive and she improved quickly following administration of fluids and supplemental oxygen. She received 2 doses of betamethasone for fetal indications. However, we now know corticosteroids are beneficial in those with COVID-19 requiring oxygen supplementation and may have had a secondary beneficial effect on maternal condition. 
- Acute kidney injury (AKI) is commonly reported with COVID-19 [9] and has been seen in transplanted patients with COVID-19 [6]. AKI is felt to be multifactorial in most cases, although direct viral effects on the kidney have been reported [9]. In this case the rise in creatinine was mild and likely pre-renal in aetiology.

- Management of kidney transplant recipients during pregnancy is often challenging, with high rates of preeclampsia being reported, and the emergence of COVID19 has added an extra layer of complexity. In this case, rapid diagnosis, supportive care and a measured multidisciplinary team (MDT) approach involving obstetrics, obstetric physicians and nephrology resulted in complete recovery from a respiratory standpoint and delivery of a healthy baby at term. We need to continue to be mindful of the risks of COVID-19 exposures as women travel to hospital for face-to-face visits, blood tests and scans and weigh these against monitoring a high risk pregnancy. If a kidney transplant recipient develops COVID-19 during pregnancy a personalised approach to supportive management, delivery and immunosuppression must be taken.

\section{Compliance with ethical standards}

Conflict of interest The authors of this manuscript have no conflicts of interest to disclose as described by the Journal of Nephrology.

Ethics approval The case report for this 'lessons for the clinical nephrologist' paper was written with the consent of the patient

\section{References}

1. Rasmussen SA, Smulian JC, Lednicky JA, Wen TS, Jamieson DJ (2020) Coronavirus Disease 2019 (COVID-19) and pregnancy: what obstetricians need to know. Am J Obstet Gynecol 222(5):415-426

2. Gynaecologists RC of O\&. Coronavirus (COVID-19) infection in pregnancy. Inf Healthc Prof Version. 2020;4.

3. Ellington S (2020) Characteristics of women of reproductive age with laboratory-confirmed SARS-CoV-2 infection by pregnancy status-United States, January 22-June 7, 2020. MMWR Morb Mortal Wkly Rep [Internet];69. Available from: https://www.cdc. gov/mmwr/volumes/69/wr/mm6925a1.htm. Accessed 2 June 2020

4. Knight M, Bunch K, Vousden N, Morris E, Simpson N, Gale C, et al. (2020) Characteristics and outcomes of pregnant women admitted to hospital with confirmed SARS-CoV-2 infection in UK: national population based cohort study. BMJ. m2107.

5. Zhu L, Gong N, Liu B, Lu X, Chen D, Chen S et al (2020) Coronavirus Disease 2019 pneumonia in immunosuppressed renal transplant recipients: a summary of 10 confirmed cases in Wuhan China. Eur Urol 77(6):748-754

6. Banerjee D, Popoola J, Shah S, Ster IC, Quan V, Phanish M (2020) COVID-19 infection in kidney transplant recipients. Kidney Int 97(6):1076-1082

7. British Transplant Society. Guidance on the management of transplant recipients diagnosed with or suspected of having COVID19.

8. Thangaratinam S, Khan R, Blott M (2020) Guidance for maternal medicine services in the evolving coronavirus (COVID-19) pandemic.

9. Ronco C, Reis T, Husain-Syed F (2020) Management of acute kidney injury in patients with COVID-19. Lancet Respir Med. S2213260020302290.

Publisher's Note Springer Nature remains neutral with regard to jurisdictional claims in published maps and institutional affiliations. 